\title{
Corrección quirúrgica de la anquilosis unilateral de la articulación temporomandibular. Reporte de caso
}

\author{
Surgical correction of unilateral ankylosis of the temporomandibular joint; Case \\ report
}

Kemberly Gabriela Viera ${ }^{1 a}$, Víctor Alexander Cruz ${ }^{1 a}$, Michael Xavier Quisilema ${ }^{\text {bc }}$ Angélica Maria Jaimes ${ }^{a c}$

\begin{abstract}
Resumen
La anquilosis unilateral de la articulación temporomandibular corresponde a un trastorno temporomandibular clasificado dentro de la hipomovilidad mandibular crónica. El objetivo del presente caso clínico, proporcionar una guía tanto clínica como diagnostica sobre esta patología, para la comunidad odontológica, brindando una nueva alternativa para que se puede corregir dicha anomalía y de la mano mejorar la calidad de vida de nuestro paciente, con un tratamiento cien por ciento científico, profesional y garantizado. La literatura define anquilosis como una inmovilidad anormal de la mandíbula, desorden que lleva a una restricción de la apertura bucal con reducción parcial de los movimientos mandibulares o una completa inmovilidad de la mandíbula. Presentamos un caso de anquilosis temporomandibular unilateral (izquierda) en un niño de 14 años, que tratamos con resección del bloque anquilótico, tras fractura mandibular con la finalidad de elongar los tejidos blandos y mantener el espacio en la nueva cavidad glenoidea para evitar la re-anquilosis y favorecer la remodelación del cóndilo.
\end{abstract}

Palabras Claves: anquilosis; articulación temporomandibular; Cóndilo Mandibular. (Fuente: DeCS BIREME)

\section{ABSTRACT}

The unilateral ankylosis of the temporomandibular joint corresponds to a temporomandibular disorder classified within the chronic mandibular hypomobility. The objective of the present clinical case, to provide a clinical and diagnostic guide on this pathology, for the dental community, providing a new alternative so that this anomaly can be corrected and hand improve the quality of life of our patient, with a treatment One hundred percent scientific, professional and guaranteed. The literature defines ankylosis as an abnormal immobility of the jaw, disorder that leads to a restriction of the buccal opening with partial reduction of mandibular movements or a complete immobility of the jaw. We present a case of unilateral temporomandibular ankylosis (left) in a 14-year-old boy, who treated with ankylosing block resection, after mandibular fracture in order to elongate the soft tissues and maintain space in the new glenoid cavity to avoid re-ankylosis and favor the remodeling of the condyle.

Key-words: ankyloses; temporomandibular joint; Mandibular Condyle . (Source: MeSH NLM)

\footnotetext{
${ }^{1}$ Universidad Tecnológica Equinoccial

a Odontólogo

b Doctor en Estomatología

${ }^{\circ}$ Cirujano Maxilofacial
}

Correspondencia:

Víctor Alexander Cruz

Correo electrónico: alexandercruz1993@hotmail.com
Este es un artículo Open Access distribuido bajo la licencia Creative Commons Atribución-NoComercialCompartir Igual 4.0

Citar como: Viera K, Cruz V, Quisilema M, Jaimes A. Corrección quirúrgica de la anquilosis unilateral de la articulación temporomandibular; Reporte de caso. KIRU. 2019; abril-jun; 16(2): 75-80. https://doi.org/10.24265/kiru.2019.v16n2.04 


\section{INTRODUCCIÓN}

La anquilosis de la articulación temporomandibular (ATM) es la limitación o bloqueo de la de la misma, la cual puede ser parcial o total, uno o bilateral, fibrosa u ósea. El objetivo del presente caso clínico, proporcionar una guía tanto clínica como diagnostica sobre esta patología, para la comunidad odontológica, brindando una nueva alternativa para que se puede corregir dicha anomalía y de la mano, mejorar la calidad de vida de nuestro paciente, con un tratamiento cien por ciento científico, profesional y garantizado. Es una de las más serias e incapacitantes condiciones patológicas que pueden ocurrir en la región máxilo facial porque interfiere en el habla, masticación, higiene oral y en el crecimiento facial y mandibular. Puede ser clasificada, de acuerdo con el local de ocurrencia en, intra capsular o extra capsular; conforme el tipo de tejido envuelto, o sea, tejido óseo, tejido fibroso o tejido fibro-óseo y de acuerdo con su extensión en completa (donde la articulación está totalmente fundida) o incompleta (donde no hay fusión total). Puede envolver solamente el cóndilo, solamente el proceso coronoide o ambos ${ }^{(1,2)}$.

El término "anquilosis" en griego se refiere a una soldadura u atadura, que en el caso del complejo de la articulación temporomandibular, representa una adhesión ósea o fibrosa que conlleva a la pérdida progresiva de la función. La adhesión puede ocurrir entre la parte más superior de la apófisis condilar y la fosa glenoidea del hueso temporal o también entre cualquier tejido duro o blando del hueso mandibular y el hueso maxilar, hueso cigomático o base de cráneo (2).

La anquilosis de la articulación temporomandibular puede ser: verdadera cuando el daño es causado intraarticularmente por la adhesión del cóndilo mandibular a la cavidad glenoidea y falsa o pseudo-anquilosis, cuando las estructuras extra- articulares son la causa de la hipomovilidad mandibular, dentro de las que podemos citar a la hiperplasia de la apófisis coronoides, fracturas no reducidas del arco cigomático, fibrosis post-radiación del músculo temporal y desórdenes neurológicos, entre otros ${ }^{(3)}$

Esta entidad puede ser clasificada mediante combinaciones terminológicas refiriéndose a la localización (intra o extra articulares), tipo de tejido involucrado (ósea, fibrosa o fibro-ósea), extensión de la fusión (completa o incompleta) y lado de localización (unilateral, bilateral) ${ }^{(2,3)}$.

Realizando revisiones de la literatura en cuanto a agentes etiológicos encontramos una alta asociación con los traumatismos maxilofaciales, enfermedades sistémicas dentro de las que se destacan: espondilitis anquilosante, artritis reumatoide y la psoriasis, teniendo en cuenta también las infecciones locales o sistémicas con repercusión articular ${ }^{(4)}$.

El diagnóstico de la anquilosis témporomandibular se da a través de la evaluación clínica y exámenes complementarios como las radiografías panorámicas, transcreneanas, posteroanterior de mandíbula, telerradiografías de perfil y otros métodos más modernos de diagnóstico por imagen, tales como tomografía computadorizada y resonancia magnética ${ }^{(5,6,3)}$.

El objetivo del presente caso clínico, proporcionar una guía tanto clínica como diagnostica sobre esta patología, para la comunidad odontológica, brindando una nueva alternativa para que se puede corregir dicha anomalía y de la mano mejorar la calidad de vida de nuestro paciente, con un tratamiento cien por ciento científico, profesional y garantizado. El tratamiento de la anquilosis de la articulación temporomandibular debe ser quirúrgico asociado a la interposición de algún material en el espacio formado tras la realización de la condilectomía. Ese material puede ser autógeno o aloplástico para que se evite la recidiva (7). La anquilosis de la articulación temporomandibular, cuando diagnosticada precozmente, tiene pronóstico favorable debido al crecimiento óseo de la mandíbula que todavía continúa de forma normal en niños y algunos adolescentes. Se debe también tras la cirugía encaminar al paciente para la fisioterapia oral, pues esta se hace necesaria para la completa recuperación fisiológica del movimiento de la mandíbula, evitando la re-anquilosis de la articulación temporomandibular ${ }^{(8)}$.

\section{REPORTE DEL CASO}

Paciente masculino de 14 años de edad, raza mestiza, y nacionalidad ecuatoriana acude a Cirugía y Traumatología Buco-Maxilo-Facial en el hospital "Pablo Arturo Suarez", en la Ciudad de Quito-Ecuador, por presentar dificultad para abrir y cerrar la boca lo que le dificulta alimentarse, además presenta, dolor a nivel de la zona temporal del lado izquierdo con 6 años deevolución y con un estado óptimo de salud aparentemente. Tras la firma del consentimiento informado por parte de su represéntate legal (MADRE) autorizando su análisis, tratamiento y posterior difusión en relato de caso clínico, respetando los principios de ética.

Un dialogo y análisis inicial permitió conocer que hace 6 años atrás, la paciente inicio con la molestia después de presentar un traumatismo en la zona, la cual no fue atendida, porque no presento molestias mayores, hasta que luego de varios años, empezó a presentar molestia y dificultar a la hora de ingerir alimentos lo cual, hace que la familia acuda al centro de especialidades para su diagnóstico, atención, tratamiento y recuperación. 
En la historia médica, el paciente no refiere patología actual, ni tampoco antecedentes familiares o personales de importancia. Al examen extraoral, la paciente no presenta ninguna patología aparente, sin embargo, a la palpación presenta dolor en el área temporal del lado izquierdo, además presenta una dificultad marcada a la hora de una apertura bucal máxima. Al examen intraoral se observa una dentición definitiva completa (con ausencia de terceros molares), un buen estado salud oral, sin presencia de caries dental. Los tejidos gingivales normales y ausencia de placa y cálculo dental. Se constante previo a la cirugía, mediante una fotografía extraoral de frente, la condición actual del paciente y la limitada apertura bucal. (Figura 1)

Además, como examen complementario se realizó una tomografía axial computarizada, de corte coronal previo al acto quirúrgico, para un mejor estudio del área. (Figura2)

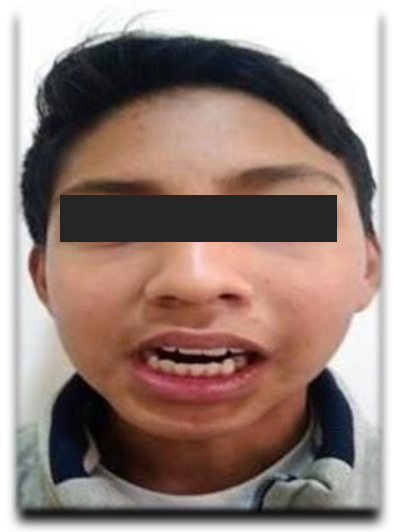

Figura 1. Fotografía extraoral de frente, se observa una apertura bucal limitada de aprox. 1,5 cm. El paciente manifestaba ligero dolor a la apertura bucal máxima

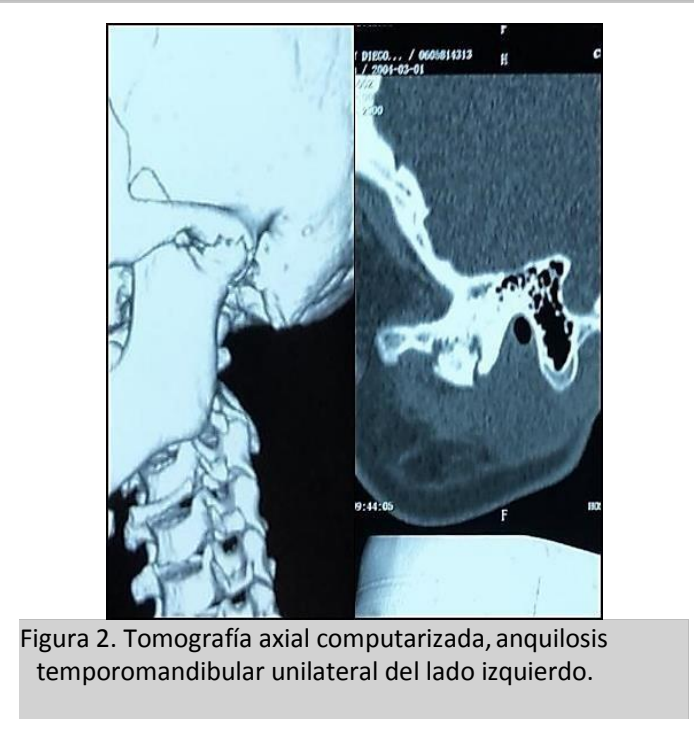

Se planificó la intervención quirúrgica, previa a todo el análisis y diagnóstica por parte del Cirujano, contando con el consentimiento de su tutor legal.

El acto quirúrgico comenzó con la intubación nasotraqueal, la cual se efectuó con fibroscopio. Se realizó un abordaje preauricular el cual tiene nos proporciona una ventaja importante, ya que es directa y permite una visualización amplia y correcta, posteriormente la inserción se practica delante del oído extendiéndola desde sus inserciones superiores a las inferiores y que siga el trayecto contorneando el tragus de
$5 \mathrm{~mm}$ aproximadamente. Se separó el colgajo cutáneo de aproximadamente $2 \mathrm{~mm}$ de espesor por delante de la incisión. Se realizó la palpación del cóndilo y se procede a la disección completa del mismo, para postreramente colocar el injerto proveniente del tragus. Se reseco el cóndilo por debajo de su borde superiora unos $10 \mathrm{~mm}$, esto se lleva a cabo mediante una fresa quirúrgica redonda o cilíndrica. De esta manera se va a crear una nueva cavidad glenoidea. Posteriormente se realizó un control de la hemorragia. En el transcurso de la cirugía se procedió a la extirpación de la bolsa adiposa de bichat. Para terminar con el acto quirúrgico se procedió a la 
sutura (000) por planos con la finalidad de elongar el tejido blando y prevenir la re-anquilosis, manteniendo separadas las superficies cruentas de la resección. Se aplicó un apósito compresivo sobre el odio y se deja en su sitio durante 48 horas.
Se indica al paciente que utilice la articulación tan pronto como le sea posible. Tanto el postoperatorio inmediato y tardío fueron vigilados consuntamente por el cirujano, con la medición interincisal anterior de la apertura bucal. (Figura 3)

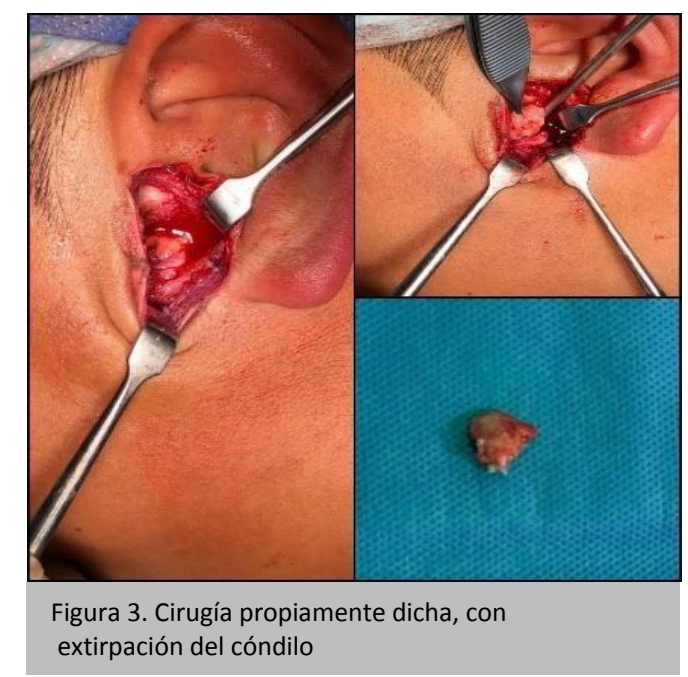

Se indica al paciente que luego de que el dolor postoperatorio ha disminuido se haga ejercicios de apertura y cierre bucal, con ayuda de goma de mascar.

Esta fase de recuperación tiene como objeto lograr que la apertura bucal obtenida con la cirugía no se reduzca. El tratamiento se inicia con fisioterapia inmediata y agresiva después de 24 horas de la cirugía. Cuando el dolor postoperatorio ha disminuido, se indica al paciente que realice movimientos de apertura y cierre durante 20 minutos cuatro o cinco veces al día. En la cual va a utilizar goma de mascar sin azúcar entre cada ejercicio.

Como complemento se realiza una interconsulta con el ortodoncista para que una vez terminado el periodo aguado de recuperación, y con un paciente más colaborador, y motivado, empiece a utilizar un tipo especial de aparatología funcional. Se continúa con la fisioterapia, para estimular el crecimiento y remodelación de la rama, utilizando el Spring Bite, que estimula la apertura y cierre y favorece la rotación mandibular. Los dispositivos a usar se denominan pistas planas asimétricas. Esto se empezar a usar después de una semana del tratamiento quirúrgico y se utilizara durante 20 minutos cuatro veces al día. Asimismo, se intercalan los ejercicios de la etapa anterior para que la fisioterapia sea aún más vigoro.

El paciente acude a la consulta después de una semana del acto quirúrgico sin ninguna novedad, con un aumento marcado en la apertura bucal, y listo para empezar con el uso de la aparatología funcional con el objetivo de evitar algún tipo de recidiva. Figura 4. A y $B$

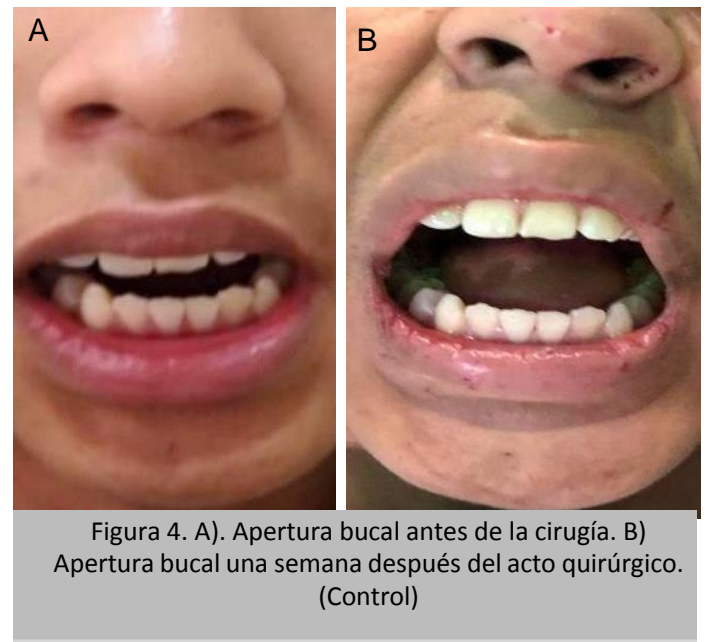




\section{DISCUSIÓN}

La anquilosis temporomandibular es una alteración en la infancia que causa graves trastornos funcionales, orgánicos y de armonía facial. Y una de las causas más frecuentes y con un alto porcentaje son los traumatismos automovilísticos, caídas, heridas por proyectil dearma de fuego, accidentes laborales; son factores etiológicos capaces de producir daño articular y desencadenar la formación de tejido óseo o fibroso que ocasionan anquilosis. Otra etiología menos frecuente son las infecciones recurrentes del conducto auditivo interno, artropatías sistémicas o lesiones tumorales ocupan también un lugar importante en las causas que provocan esta patología $(5,6)$.

La anquilosis puede ser uni o bilateral, presentarse durante o después del desarrollo mandibular, pudiendo afectar a mujeres y hombres por igual. Los pacientes suelen acudir a la consulta en busca de tratamiento años después de presentarse la etiología desencadenante, refiriendo incapacidad progresiva para abrir la boca. Al interrogatorio es invariable encontrar antecedentes de traumatismo facial no tratado en su momento (fracturas condilares). No es raro que el paciente refiera también cierto grado de obstrucción respiratoria sobre todo al dormir ${ }^{(7)}$.

El interrogatorio, examen físico y radiográfico son de gran importancia para establecer etiología, diagnóstico y plan de tratamiento adecuado. A todo paciente que requiera condilectomía, previamente a la cirugía se le tiene que sensibilizar y hacer que tome conciencia de la magnitud del problema y así evitar otra intervención por reanquilosis. Por otro lado, también es claro que cuando la patología es en el cóndilo, la intervención debe ser a nivel del cóndilo y no alejada de este, donde lo único que se logrará es que el paciente continúe con el problema condilar (5-7).

El diagnóstico se basa en la experiencia clínica para reconocer los signos y síntomas y en el uso de métodos auxiliares de diagnóstico: radiografías simples, la tomografía computada con reconstrucción tridimensional, la resonancia magnética y la esterolitografía para apoyar el diagnóstico y brindar al paciente un tratamiento completo y ajustado a su caso en particular. ${ }^{(8)}$

Es esencial un programa terapéutico para la correcta regeneración del cóndilo. Si se restaura la oclusión y la función se normaliza, la superficie articular puede regenerarse y remodelarse. ${ }^{(8)}$

En el caso de ser unilateral se presenta desviación al lado afectado, desviación de la línea media, inclinación del plano oclusal y acortamiento de la rama anquilosada; hipertrofia de los músculos maseteros y disminución de los movimientos de lateralidad; un crecimiento vertical excesivo del lado no afectado y alteración en la oclusión, como mordida cruzada o telescópica. En los casos bilaterales se produce una retrognasia importante, un exceso vertical anterior (sonrisa de encía), con diferentes grados de hipoplasia del mentón ${ }^{(9)}$.

La anquilosis es la fusión total o parcial de los componentes de una articulación. En la anquilosis temporomandibular se fusionan el cóndilo mandibular, la fosa glenoidea y la eminencia articular del hueso temporal, lo que impide la apertura bucal y limita los movimientos mandibulares normales. Esto interfiere con el crecimiento y desarrollo de la mandíbula, provocando graves trastornos funcionales, orgánicos y alterando la armonía facial. De ahí la importancia de un diagnóstico y tratamiento quirúrgico oportunos ${ }^{(10)}$.

La anquilosis temporomandibular puede deberse a un traumatismo craneoencefálico, facial, principalmente mandibular sobre todo del mentón. Los pacientes jóvenes sufren con mayor frecuencia fractura del cuello del cóndilo generalmente en tallo verde. Si no se diagnosticano si se tratan en forma incorrecta se produce anquilosis, principalmente en niños menores de diez años de edad (11).

La fisioterapia precoz es esencial en estos pacientes. La ventaja que ofrece esta técnica respecto a las que requieren la utilización de un bloqueo intermaxilar es la posibilidad de iniciar los ejercicios de rehabilitación inmediatamente después de la cirugía ${ }^{(12)}$.

\section{CONCLUSIONES}

La anquilosis de la articulación temporomandibular es un problema poco común pero que afecta la funcionalidad de la misma, la cual acarrea asimetrías faciales que compromete la estética de los pacientes tanto en niños/as y adolescentes. Lamentablemente la etiología más frecuente corresponde a las secuelas de traumatismos maxilofaciales que provocan fracturas mandibulares con tratamientos ausentes o mal planteados, además de la osteomielitis de origen ótico, dérmico y odontogénico. El tratamiento planteado en nuestro servicio consiste en liberar el bloque anquilótico a nivel de la articulación temporomandibular, muchas veces ya irreconocible, para que de esta manera recuperemos una funcionalidad correcta o estable la cual será proporcionada en base a una pseudoartrosis uni o bilateral que no comprometa la salud articular. Es de suma importancia el manejo de estos pacientes, el control y la fisioterapia agresiva conseguida por la confección de una apertura bucal sin olvidar el seguimiento en el tiempo edades oportunas para corregir las secuelas ocasionadas por esta patología. 
Contribuciones de autoría: KGV, VAC, MXQ y AMJ diseñaron el estudio, recopilaron, analizaron los datos. Redactaron y aprobaron el artículo todos los autores.

Fuente de financiamiento: El estudio fue financiado por los autores.

Conflicto de intereses: Los autores declararon no tener conflictos de interés.

\section{REFERENCIAS}

1. Atilas $A$, Costa $A$, Dominguete $P$, Andrade $G$, Generoso $\mathrm{R}$, Ribeiro A. Anquilosis condilar mandibular unilateral - relato de caso clínico. Acta odontol. venez [Internet]. 2008 [citado 01/03/2019] ;46(2):187-190. Disponible en: http://www.scielo.org.ve/scielo.php?script=sci_arttext\& pid $=S 0001-63652008000200016 \& \operatorname{lng}=e s$

2. Broggi A, Cabrera G, Caballero M, Oré A. Manejo contemporáneo de la Anquilosis de la Articulación Temporomandibular en el niño y el adolescente: Reporte de Casos. Acta méd. Peruana [Internet]. 2013 [citado 01/03/2019];30(2): 86-91. Disponible en: http://www.scielo.org.pe/scielo.php?script=sci_arttext \& pid=S1728-59172013000200007\&lng=es.

3. Bello S, Olokun B, Olaitan A, Ajike S; Aetiology and presentation of ankylosis of the temporomandibular joint: report of 23 cases from Abuja, Nigeria. Brit J Oral Max Surg. 2012; 50(1): 80-84.

4. Qudah M, Qudeimat M, Al-Maaita J; Treatment of TMJ ankylosis in Jordanian children - a comparison of two surgical techniques. J Cranio Maxill Surg.2005; 33(1): 30-36.
5. Morey M, Caubet J, Iriarte I, Quirós P, Pozo A, Forteza I. Tratamiento de anquilosis tempomandibular en la infancia mediante artroplastia y distracción de tejidos blandos. Rev Esp Cirug Oral y Maxilofac [Internet].2004 [citado 01/03/2019]; 26(4):240-244. Disponible en: http://scielo.isciii.es/scielo.php?script=sci_arttext\&pid= S1130-05582004000400004\&lng=es.

6. Molina D, Aguayo P, Ulloa C, Iturriaga V, Bornhardt T, Saavedra M. Anquilosis de la articulación temporomandibular: una revisión de la literatura. Av Odontoestomatol [Internet]. 2013 [citado 01/03/2019]; 29( 5 ): 239-244. Disponible en: http://scielo.iscii.es /scielo.php?script=sci_arttext\&pid=S02131285201300 0500003\&lng=es.

7. Pereira A, Aleva N. Anquilose da articulação temporomandibular: uma nova proposta para tratamento. Revista odonto ciencia. 1996;21(1):67-74.

8. DeFabianis P. TMJ fractures in children and adolescents: treatment guidelines. J Clin Pediatr Dent. 2003;27(3):191.

9. De Fabianis P. Treatment of condylar fractures in children and youths: the clinical value of the occlusal plane orienta-tion and correlation with facial development (case reports). J Clin Pediatr Dent 2002;26(3):243-50.

10. Kimura FT. Resultados del tratamiento quirúrgico de 52 casos de anquilosis temporomandibular. Cir Ciruj 2003;71(1):12-21.

11. Ahmad QG, Siddiqui RA, Khan AH, Sharma SC. Interposition arthroplasty in temporomandibular joint ankylosis. Indian J Otolaryngol Head Neck Surg 2004;56(1):5-8.

12. Maccari-filho $M$, Palombo $C$, Maccar. Ancilose congênita temporomandibular - um caso extremo, Rev. Ciências Med da PUC/Campinas, 1998; (7(2); 1 - 12. 